\title{
6. Alken-Preisträgertreffen 2001
}

PD Elmar Gerharz erhält Auszeichnung

Das Alken-Preisträger-

treffenz2001.fand vom

29. November bis

1. Dezember 2001 in

München statt und wurde von PD Hansjörg Danuser, Bern, organisiert. Der C.E. Alken-Preis wurde PD Elmar Gerharz, Würzburg, für seine Arbeit über die Auswirkungen von Urinableitungen auf Knochenstruktur und den Stoffwechsel verliehen. Die folgende Zusammenfassung soll eine kurze Übersicht über die breitgefächerten Referate geben.
Prostata- und Blasenkarinom

Prof. Urs E. Studer, Bern, sprach als Gastreferent über die Bedeutung einer ausgedehnten Lymphadenektomie bei Blasenund Prostatakarzinomen. Bei 452 Patienten mit invasivem Urothelkarzinom der Harnblase, die im präoperativen Staging als lymphonodal negativ galten (CT-Abdomen-Becken) fand man bei 83 Patienten $(18 \%)$ dank ausgedehnter Lymphadenektomie mikropathologisch sowohl ipsi- als auch kontralateral positive Lymphknoten. Dabei zeigten diejenigen Patienten eine signifikant bessere 5-Jahres-Überlebensrate mit Lymphknoten-Durchmesser $<0,5 \mathrm{~cm}$, ohne extranodales Wachstum und mit niedrigem T-Stadium. 30\% der Patienten mit mikroskopischen Lymphknotenmetastasen leben noch nach 5 Jahren.

Bei 333 Patienten mit einem Prostatakarzinom im präoperativen Staging (CT-Abdomen-Becken) lymphonodal negativ, fanden sich 77 Patienten (23\%) mit histologisch positiven Lymphknoten. Dabei verdoppelte sich die Anzahl der nachgewiesenen karzinombefallenen Lymphknoten dank der Ausdehnung der Lymphadenektomie. Hätte man auf die ausgedehnte Lymphadenektomie verzichtet, wären $20 \%$ aller Patienten im pN Stadium „understaged“ gewesen. Die Daten zeigen zudem, dass bei Prostatakarzinompatienten mit PSA > $10 \mathrm{mg} / \mathrm{l}$ und/oder Gleason Score $>6$ eine ausgedehnte Lymphadenektomie durchgeführt werden soll, womit die Überlebensprognose möglicherweise verbessert wird.
Als Gastreferent berichtete Prof. Werner Hohenberger, Erlangen-Nürnberg, über seine Erfahrungen mit der nervenerhaltenden chirurgischen Technik bei operativer Behandlung von Rektumkarzinomen. Er konnte dabei zeigen, dass eine Plexus pelvicus-erhaltende Operationstechnik die Radikalität des Eingriffs nicht beeinträchtigt. Deutlich verbessert werden konnten dagegen die funktionellen Ergebnisse bei nervenerhaltender Technik bezüglich Blasenentleerungsstörungen und erektiler Dysfunktion. Zusätzlich kann die Rezidivrate beim kolorektalen Karzinom nach totaler mesorektaler Exzision durch eine präoperative, neoadjuvante Radio-/Chemotherapie gesenkt werden, ohne dass der nachfolgende operative Eingriff mit einer erhöhten postoperativen Morbidität behaftet ist. Interessanterweise konnten nach neoadjuvanter Therapie auch beinahe doppelt so viele Patienten sphinktererhaltend operiert werden. Die Tatsache, dass die Rezidivrate auch von der Technik des operierenden Chirurgen abhängt, ruft nach einer Qualitätskontrolle.

Dr. J. W. Melchior, Mainz, stellte 2 neue, computererrechnete Inhibitionsmoleküle der Histon-Deacetylase vor, nämlich CG 1255 und CG 1521. Dabei fand sich eine dosisabhängige Hemmung der Histon-deacetylase in verschiedenen Tumorzelllinien, so z.B. in LNCaP, BM1604, PC-3 und CU 145. Die dabei nötige Substanzkonzentration war im Vergleich mit Phenylbutyrat 70x (CG1255) resp. 4000x (CG1521) geringer. Durch diese beiden Moleküle wird der Zellzyklus in der 
der Patienten (18\%) wurden dabei mittels nervenerhaltender Technik operiert. Der Fragebogen wurde von 289 Patienten (72\%) ausgefüllt. 202 Patienten (70\%) waren absolut kontinent mit einem hohen Zufriedenheitsgrad. Insgesamt 41 Patienten $(14 \%)$ waren mit dem Resultat der Operation nicht zufrieden, fnehrheitlich wegen postoperativ erektiler Dysfunktion. 36 von 53 Patienten (63\%), welche nervenerhaltend operiert wurden, berichteten über suffiziente Erektionen, 4 von 36 mit Hilfe von Sildenafil. Interessanterweise wiesen die Patienten, welche nervenschonend operiert wurden, auch bessere Resultate hinsichtlich generellem Wohlbefinden, IPSS-Score und Kontinenz auf.

\section{Nierenzellkarzinome}

Prof. Karl-Heinz Kurth, Amsterdam, präsentierte Studienergebnisse der M-VACSWOG-Studie bei invasivem Blasenkarzinom. Bei Patienten mit invasivem Urothelkarzinom der Harnblase pT2-4 ( $\mathrm{n}=$ 298) konnte für die Gruppe Zystektomie + M-VAC vs. alleinige Zystektomie ein 5 Jahres-Überlebensvorteil von 57 vs. $42 \%$ gezeigt werden. Das durchschnittliche Überleben betrug 74 vs. 43 Monate zugunsten von Patienten mit Zystektomie/M-VAC. Offenbar wurden jedoch vor allem Patienten mit einem fortgeschrittenen Karzinom in die adjuvante Gruppe rekrutiert, was zu einem Selektions-Bias für diese Gruppe führte. Zudem verschwand der signifikante Überlebensvorteil bei Analyse mittels double sided tests, so dass diese Resultate keine definitive Aussage erlauben. Ob eine adjuvante Chemotherapie tatsächlich zu einem Überlebensvorteil führt, bleibt weiterhin unklar und ist Gegenstand einer aktuellen EORTC-Studie. Interessanterweise fand man in einer Subgruppenanalyse nach Berücksichtigung des Selektionsbias ausschließlich einen Überlebensvorteil für Patienten, welche möglichst radikal lymphadenektomiert wurden.

Dr. Thomas Reckwitz, Dortmund, untersuchte mittels Fragebogen die Lebensqualität nach radikaler Prostatektomie bei 401 Männern mit einem durchschnittlichen Follow-up von 3 Jahren. 53 tastasierenden Nierenzellkarzinomen. Dabei wurden möglichst alle intraperitonealen Metastasten chirurgisch entfernt. Ein Nichtwiederauftreten von Aszites wurde bei 78\% der Patienten beobachtet, weniger Schmerzen traten bei $88 \%$ auf und einen verbesserten WHO-Performance Status zeigten $78 \%$ der Patienten.
Konnte keine radikale Peritonektomie erzielt werden, waren die Resultate deutlich schlechter. So scheint zumindest in diesem relativ kleinen Kollektiv die radikale Peritonektomie bei sonst ausbehandelten Patienten eine therapeutische Option darzustellen.

Urodynamik und Neurourologie

Dr. Chris Constantinou, Standford/USA, untersuchte an 39 gesunden Probanden das Miktionsverhalten im zirkadianen Rhythmus in Abhängigkeit der täglichen Trinkmenge. Er fand dabei, dass eine Verdoppelung der Trinkmenge auf $30 \mathrm{ml} / \mathrm{kg}$ $\mathrm{KG} /$ Tag erwartungsgemäß die Urinproduktion und Miktionsfrequenz erhöhte, aber auch zu einer Zunahme des Urethraöffnungsdruckes, des maximalen Detrusordruckes, der Blasenkontraktilität und der Blasenkapazität führt. Somit sind gemessene urodynamische Parameter von der täglichen Urinproduktion abhängig, was bei der Interpretation dieser Parameter berücksichtigt werden soll.

PD Markus Hohenfellner, Mainz, referierte über seine Langzeiterfahrungen mit der sakralen Neurostimulation bei 27 Patienten mit neurogener Blasenfunktionsstörung. Bei 12 von 27 Patienten konnte nach erfolgreicher perkutan-sakraler Teststimulation ein permanenter sakraler Neurostimulator unilateral an die dorsale S3-Wurzel implantiert werden. Bei einem Patienten musste der Stimulator wegen Wundinfekt wieder entfernt werden. 3 Patienten hatten keine Verbesserung der Symptomatik. Bei den restlichen 8 Patienten wurde die Blasenfunktionsstörung um mindestens 50\% während durchschnittlich 54 (11-96) Monaten verbessert. Danach waren 7 von 8 Implantaten nicht mehr effektiv. Der Autor plädiert aufgrund dieser enttäuschenden Resultate für eine Verbesserung der Stimulationstechnik durch eine bilaterale, direkte Stimulation der sakralen Nervenwurzeln.

Harnableitungen

Prof. Tillmann Kälble, Fulda, berichtete anhand einer Literatursuche über das Tumorrezidivrisiko bei verschiedenen For- 
men der Harnableitung. Dabei fand er 56 beschriebene Rezidivtumoren, 25 davon in Harnableitungen mit Koloninterponat nach einer mittleren Latenz von 11 Jahren und 31 Rezidive bei Verwendung von Ileum. Die mittlere Latenz bis zum Auftreten eines Tumors war bei maligner Vorerkrankung 16 (7-18) Jahre und bei benigner Vorerkrankung 22 (0,3-28) Jahre. Bei Kolonersatzblasen trat in $60 \%$ und bei ilealen Ersatzblasen in 40\% der Tumor im Bereich der ureterointestinalen Anastomose auf. Der Referent empfiehlt deshalb, ab dem 5. postoperativen Jahr die regelmäßige endoskopische Kontrolle der Urinableitung.

Prof. Rudolf Hohenfellner, Mainz, stellte die Resultate der Ureterimplantation bei 651 Patienten mit Mainz Pouch I vor. Dabei fand sich in der Nachsorge bei $47 \mathrm{~Pa}$ tienten $(7,2 \%)$ eine Ureterstenose auf Höhe der antirefluxiven Ureterimplantationsstelle, welche einer Reimplantation oder seltener einer Dilatation bedurfte. Die Stenoserate war dabei bei den Patienten mit adjuvanter Strahlentherapie doppelt so hoch wie bei Patienten ohne Strahlentherapie. In dem untersuchten Kollektiv gab es 2 Häufigkeitsgipfel des Auftretens einer Implantationsstenose, nämlich nach 2-3 respektive 6-7 Jahren. Aufgrund dieser Ergebnisse und der Erkenntnis, dass nicht antirefluxiv implantierte Ureteren zu keinem relevanten Reflux und insbesondere keiner Nierenfunktionsverschlechterung führen, wurde die offene Technik der Ureterimplantation ohne Antirefluxplastik propagiert.

Prof.J. W. Thüroff, Mainz, stellte eine vereinfachte Technik der orthotopen ileozökalen Ersatzblase vor. Bei 16 Patienten wurde mittels Staplertechnik das Ileum auf das Zökum anastomosiert und die Ureteren direkt in das terminale Ileum implantiert. Die dazwischenliegende Ileozökalklappe soll dabei als Antirefluxklappe dienen. Es traten keine größeren chirurgischen Komplikationen auf. 1 von 33 Patienten musste wegen Ureterstenose reoperiert werden. Die Kontinenz tags und nachts als auch die Blasenentleerung sind gut. Die Operationszeit konnte mit dieser modifizierten Mainz-Pouch I-Technik um durchschnittlich 30 Minuten verkürzt werden. Langzeitergebnisse liegen zurzeit noch nicht vor.

PD Elmar Gerharz, Würzburg, berichtete in seinem Preisträgerreferat über Ergebnisse bei je 30 Ratten mit ilealer Ersatzblase, ilealer Ersatzblase mit Ileozökalresektion oder Kolonersatzblase; 30 Ratten ohne Operation dienten als Kontrollgruppe. Alle Gruppen zeigten eine normale Knochenstruktur der kortikalen, für die Stabilität verantwortlichen Elemente und des linearen Knochenwachstums. Interessanterweise zeigte sich aber in den beiden Gruppen mit ilealer Ersatzblase eine deutliche Reduktion der Knochentrabekel bis zu 50\%, was insgesamt zu einer deutlichen Verschlechterung der mechanischen Eigenschaften in den Röhrenknochen führt. Bezüglich der Serumparameter PTH, Vitamin D, alkalische Phosphatase waren alle 4 Gruppen äquivalent. In beiden Gruppen mit ilealer Ersatzblase fand sich jedoch im Vergleich mit den anderen Gruppen eine Hypomagnesämie, eine konsekutive Hypokalzämie und deutlich erniedrigte Spiegel des IGF-binding Proteins 3, was auf eine gastrointestinale Malabsoprtion von Magnesium und Kalzium zurückzuführen ist und vor allem beim jungen Patienten mittel- und langfristig zu einem erhöhten Frakturrisiko führen kann. Der Preisträger folgert aus seinen Ergebnissen, dass bei älteren Patienten die Knochenstabilität und damit das Frakturrisiko wohl klinisch nicht in Erscheinung treten, jedoch beim jüngeren Patienten langfristig mit einem erhöhten Frakturrisiko gerechnet werden muss. Regelmäßige metabolische Kontrollen der Nierenfunktion, der Serumelektrolyte, insbesondere von Kalzium und Magnesium, sowie des Säure-Ba-
sen-Status sind angezeigt und bei pathologischen Zuständen zu korrigieren.

\section{Verschiedenes}

PD Peter Albers, Bonn, berichtete über seine Erfahrungen mit extensiver, radikaler Tumorchirurgie bei Patienten mit Spätrezidiven eines Hodenkarzinoms mehr als 2 Jahre nach Erstbehandlung, die üblicherweise eine ausgesprochen schlechte 5-Jahres-Überlebensrate von nur 50\% zeigen. Von den 25 Patienten mit Spätrezidiv fanden sich histologisch in $60 \%$ Teratome, in 40\% der Fälle Karzinome, die in 50-80\% auf eine Chemotherapie nicht ansprechen. Alle diese Rezidivtumoren, meist im Retroperitoneum liegend, wurden radikal entfernt. Bei Aortainvasion wurde obligat das Gefäß reseziert und durch eine Prothese ersetzt. Eine radikale Tumorexision scheint bei diesen Spätrezidiven möglich und aufgrund der schlechten Ergebnisse der Chemo-Radiotherapie, die einzige erfolgversprechende Therapiemodalität zu sein.

Prof. Christian Stief, Hannover, gelang der Nachweis der Phosphodiesterase Isoenzyme 4 und 5 in menschlichem Prostatagewebe. Durch Zugabe eines PDE-4- und PDE-5-Inhibitors war in vitro eine deutliche Relaxation der glatten Muskelfasern von Prostatagewebe möglich. Die Hemmung der Phosphodiesterase 4 und 5 könnte somit ein attraktiver neuer Ansatz für eine medikamentöse BPH-Therapie darstellen.

Prof. Hans-Udo Eickenberg, Bielefeld, konnte an 26 gesunden, mit Norethisterone hormonsupprimierten Patienten zeigen, dass durch transdermale Testosteronapplikation in Gelform (2,5\% Testosteron) ein physiologischer, konstanter Testosteronspiegel erreicht werden kann. Dabei scheint es keine Rolle zu spielen, ob das Gel bereits nach 10 Minuten wieder abgewaschen wird. Lokale Hautrötungen traten dabei bei einer geringen Anzahl der Patienten auf und verschwanden allesamt wieder. Weitere klinische Studien zur Verträglichkeit und Wirksamkeit sind geplant.

Dr. R. Gerber, PD Hansjörg Danuser, Bern 